\title{
FURTHER RESULTS ON A GENERALIZATION OF BERTRAND'S POSTULATE
}

\author{
GEORGE GIORDANO \\ Department of Mathematics, Physics and Computer science \\ Ryerson Polytechnic University \\ Toronto, Ontario, Canada M5B $2 \mathrm{~K} 3$
}

(Received November 6, 1992 and in revised form February 28, 1995)

ABSTRACT. Let $d(k)$ be defined as the least positive integer $n$ for which $p_{n+1}<2 p_{1}-k$. In this paper we will show that for $k \geq 286664$, then $d(k)<k /(\log k-2.531)$ and for $k \geq 2$, then $k(1-1 / \log k) / \log k<d(k)$. Furthermore, for $k$ sufficiently large we establish upper and lower bounds for $d(k)$.

KEY WORDS AND PHRASES. Bertrand's Postulate, Primes. 1992 AMS SUBJECT CLASSIFICATION CODE. 11N05

1. INTRODUCTION.

Let $p_{n}$ be the $n^{t h}$ prime and let $k$ be a positive integer. We define $d(k)$ to be the least positive integer $n$ for which $\mathrm{p}_{n+1}<2 \mathrm{p}_{n}-\mathrm{k}$, and consider the corresponding generalization of Bertrand's Postulate. There are other generalizations of Bertrand's Postulate, for example [1] and [2].

Dressler [3] showed that $p_{n+1}<2 p_{n}-10$ for all $n>6$. Badea [4] proved that for every integer $k \geq 1$ we have

$$
d(k) \leq\left(M_{k}+2+\left(\left(M_{k}\right)^{2}+12 M_{k}+4\right)^{1 / 2}\right) / 4
$$

where $M_{k}=\max (118,[13 \mathrm{k} / 12]+1)$. The Mathematical Review (88j:11005) of [4] points out two facts. First, "Since the prime number theorem implies $p_{n+1} \sim p_{n} \sim n \log n$ it follows that $d(k) \quad-k / \log k . "$ Second, the reviewer states that using the results found in [4] he can establish, using an elementary argument, the following: 
In this paper we will use improved upper and lower bounds for $p_{n}$ and thereby establish an improved upper bound for $d(k)$. Furthermore, we will give an explicit lower bound for $d(k)$. It is obvious that in order to establish an upper bound for $d(k)$ we would want to find conditions on $n$ which guarantee

$$
k<2 p_{n}-p_{n+1}
$$

Also in order to establish a lower bound for $d(k)$ we would want to find conditions on $n$ which guarantee

$$
k>2 p_{n}-p_{n+1}
$$

To obtain an explicit upper bound for $d(k)$ we have to observe that $k /(\log k-\log \log k)<k /(\log k-2.531)$ if $k \leq 286663$. Hence we have different upper bound functions for $d(k)$ depending upon the value of $\mathrm{k}$. Moreover, we need to use the computer languages Maple and Turbo Pascal. We use Maple to get the exact value of $\mathrm{k}$ which is needed in Lemma 7, and we use Turbo Pascal to obtain Tables 1 and 2 for small values of $k$ and provide a program to verify cases $1-3$ of Theorem 4 .

The proofs of this paper require the following results.

$$
\begin{array}{lcr}
n(\log n+\log \log n-3 / 2)<p_{n} & n \geq 2 & \text { (1.3) } \\
p_{n}<n(\log n+\log \log n-1 / 2) & n \geq 20 & (1.4) \\
p_{n}=n(\log n+\log \log n-1+0(\log \log n / \log n)) & p_{n} \leq 2.686 & 1012(1.6) \\
p_{n+1}-p_{n} \leq 652 & n \geq 28567 & (1.7) \\
\log \log (n+1)<.000003412+\log \log n & n \geq 28567 & (1.8) \\
n \log (1+1 / n)+\log (n+1)+\log \log (n+1)<.00053582 n \quad n
\end{array}
$$

(1.3) and (1.4) are found in [5], (1.5) is found in [6] and (1.6) is found in [7].

We define the following function to make Lemmas 2 and 4 more readable,

$$
\mathrm{T}(\mathrm{k}, \mathrm{c})=(1+\mathrm{c} \varepsilon) / \log \mathrm{k} \quad \text { where } \varepsilon>0 \text {. }
$$

2. THEOREMS, LEMMAS AND THEIR PROOTS.

IEMMA 1. For $n$ sufficiently large, there exists $\varepsilon>0$ such that $2 p_{n}-p_{n+1}>n(\log n+\log \log n-(1+\varepsilon))$.

PROOF. From (1.5) there exists a constant $c$ such that $p_{n}<n(\log n+\log \log n-1+c(\log \log n / \log n)$ and 
$p_{n}>n(\log n+\log \log n-1-c(\log \log n / \log n)$.

We see that

$$
\begin{aligned}
2 p_{n}-p_{n+1}>2 n(\log n & +\log \log n-1-c(\log \log n / \log n)) \\
-(n+1)\{ & \log (n+1)+\log \log (n+1)-1 \\
& +c(\log \log (n+1) / \log (n+1))\} .
\end{aligned}
$$

After simplification and for $n$ sufficiently large (2.1) will become $2 p_{n}-p_{n+1}>n(\log n+\log \log n-(1+\varepsilon))$.

QED .

IEMMA 2. With $\varepsilon$ and $\mathrm{n}$ as in Lemma 1 , let $\mathrm{n}=\mathrm{k}(1+(1+4 \varepsilon) / \log \mathrm{k}) / \log \mathrm{k}$ then for $\mathrm{k}$ sufficiently large we have $k<n(\log n+\log \log n-(1+\varepsilon))$.

PROOF. Suppose not; then

$$
k \geq n(\log n+\log \log n-(1+\varepsilon))
$$

After substituting for $n$, multiplying through by (log $\mathrm{k}) / \mathrm{k}$ and using $(1.9),(2.2)$ becomes

$$
\begin{aligned}
\log k & >\log k+\log (1+T(k, 4))-\log \log k-(1+\varepsilon) \\
& +\log \log ((k / \log k)(1+T(k, 4)))+T(k, 4) \log k \\
& +T(k, 4) \log (1+T(k, 4))-(1+\varepsilon) T(k, 4) \\
& -T(k, 4)\{\log \log k-\log \log ((k / \log k)(1+T(k, 4)))\} .(2.3)
\end{aligned}
$$

We observe that (2.3) does not hold for large $k$ because $-\log \log k+\log \log ((k / \log k)(1+T(k, 4))) \rightarrow 0$ and the $T(k, 4) \log k$ term dominates. Hence this establishes the Lemma.

QED.

THEOREM 1 . With $\varepsilon$ as in Lemma 1 , there exists $k$ sufficiently large such that $d(k)<k(1+(1+4 \varepsilon) / \log k) / \log k$.

PROOF. We want to find an upper bound for the function $d(k)$ such that for all $n \geq d(k)$ we have

$$
k<2 p_{n}-p_{n+1}
$$

For $n$ sufficiently large and $\varepsilon>0$, by Lemma 1 we have the following inequality

$$
n(\log n+\log \log n-(1+\varepsilon))<2 p_{n}-p_{n+1} .
$$

From (2.5) we now replace (2.4) with a more restrictive inequality

$$
k<n(\log n+\log \log n-(1+\varepsilon))
$$


Choose $n=k(1+(1+4 \varepsilon) / \log k)$. Then by Lemma $2,(2.6)$ and hence (2.4) still hold. Therefore $d(k)<n=k(1+(1+4 \varepsilon) / \log k) / \log k$, establishing an upper bound for $d(k)$.

QED .

LEMMA 3. For $n$ sufficiently large, there exists $\varepsilon>0$ such that $2 p_{n}-p_{n+1}<n(\log n+\log \log n-(1-\varepsilon))$.

PROOF. By using the upper and lower bounds for $p_{n}$ found in the proof of Lemma 1, we have the following

$$
\begin{aligned}
2 p_{n}-p_{n+1}<2 n(\log n & +\log \log n-1+c(\log \log n / \log n)) \\
-(n+1)\{ & \{\log (n+1)+\log \log (n+1)-1 \\
& -c(\log \log (n+1) / \log (n+1))\} .
\end{aligned}
$$

After simplification of (2.7) we have for $n$ sufficiently large the desired result $2 p_{n}-p_{n+1}<n(\log n+\log \log n-(1-\varepsilon))$ QED.

IEMMA 4. With $\varepsilon$ and $\mathrm{n}$ as in Lemma 3 , let $\mathrm{n}=\mathrm{k}(1+(1-3 \varepsilon) / \log \mathrm{k}) / \log \mathrm{k}$ then for $\mathrm{k}$ sufficiently large we have $k>n(\log n+\log \log n-(1-\varepsilon))$.

PROOF. Suppose not; then

$$
k \leq n(\log n+\log \log n-(1-\varepsilon))
$$

After substituting for $n$, multiplying through by $(\log \mathrm{k}) / \mathrm{k}$ and using $(1.9),(2.8)$ becomes

$$
\begin{aligned}
\log k \leq & \log k+\log (1+T(k,-3))-\log \log k-(1-\varepsilon) T(k,-3) \\
& +\log \log ((k / \log k)(1+T(k,-3)))+T(k,-3) \log k \\
& +T(k,-3) \log (1+T(k,-3))-T(k,-3) \log \log k-(1-\varepsilon) \\
& +T(k,-3) \log \log ((k / \log k)(1+T(k,-3))) .
\end{aligned}
$$

We observe that (2.9) does not hold for large $k$ because $-\log \log k+\log \log ((k / \log k)(1+T(k,-3))) \rightarrow 0$ and the $T(k,-3) \log k$ term dominates, thereby proving the Lemma .

THEOREM 2. With $\boldsymbol{\varepsilon}$ as in Lemma 3 , there exists $k$ sufficiently large such that $d(k)>k(1+(1-3 \varepsilon) / \log k) / \log k$.

PROOF. We want to find a lower bound for $d(k)$ such that for all $\mathrm{n}<\mathrm{d}(\mathrm{k})$ we have

$$
k>2 p_{n}-p_{n+1}
$$

For $n$ sufficiently large, Lemma 3 yields the following inequality

$$
n(\log n+\log \log n-(1-\varepsilon))>2 p_{n}-p_{n+1}
$$


From (2.11) we now replace (2.10) with a more restrictive inequality

$$
k>n(\log n+\log \log n-(1-\varepsilon)) \text {. }
$$

Choose $\mathrm{n}=\mathrm{k}(1+(1-3 \varepsilon) / \log \mathrm{k}) / \log \mathrm{k}$. Then by Lemma $4,(2.12)$ and hence (2.10) still hold. Therefore

$\mathrm{d}(\mathrm{k})>\mathrm{n}=\mathrm{k}(1+(1-3 \varepsilon) / \log \mathrm{k}) / \log \mathrm{k}$ establishing a lower bound for $d(k)$.

QED .

LEMMA 5. If $n \geq 20$, then

$2 p_{n}-p_{n+1}<n(\log n+\log \log n+1 / 2)$.

PROOF. From (1.3) and (1.4) we have

$$
\begin{array}{rl}
2 p_{n}-p_{n+1}<2 & 2 n(\log n+\log \log n-1 / 2) \\
& -(n+1)(\log (n+1)+\log \log (n+1)-3 / 2) .
\end{array}
$$

After several manipulations we see that (2.13) becomes

$$
2 p_{n}-p_{n+1}<n(\log n+\log \log n+1 / 2) \text {. }
$$

QED.

IEMMA 6. Let $\mathrm{n}=\mathrm{k}(1-1 / \log \mathrm{k}) / \log \mathrm{k}$, where $\mathrm{k} \geq 92$ then $\mathrm{k}>\mathrm{n}(\log \mathrm{n}+\log \log \mathrm{n}+1 / 2)$.

PROOF. Suppose not; then

$$
k \leq n(\log n+\log \log n+1 / 2) .
$$

After substituting for $\mathrm{n}$ and multiplying through by (log $\mathrm{k}) / \mathrm{k}$, (2.14) would become

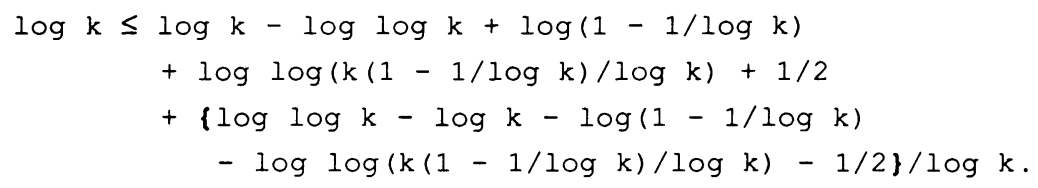

With further simplifications and rearrangement of terms we see that (2.15) is false for $k \geq 92$, thereby establishing the Lemma.

THEOREM 3. For $k \geq 2$ then $k(1-1 / \log k) / \log k<d(k)$. PROOF. We want to find a lower bound for $d(k)$ such that for all $\mathrm{n}<\mathrm{d}(\mathrm{k})$ we have

$$
k>2 p_{n}-p_{n+1}
$$

For $\mathrm{n} \geq 20$ and using Lemma 5 we establish the following

$$
2 p_{n}-p_{n+1}<n(\log n+\log \log n+1 / 2)
$$


From (2.17) we now replace (2.16) with a stronger inequality

$$
k>n(\log n+\log \log n+1 / 2)
$$

If $\mathrm{n}=\mathrm{k}(1-1 / \log \mathrm{k}) / \log \mathrm{k}$, then by Lemma $6(2.18)$ and hence (2.16) hold. Therefore $\mathrm{d}(\mathrm{k})>\mathrm{n}=\mathrm{k}(1-1 / \log \mathrm{k}) / \log \mathrm{k}$ establishing a lower bound for $d(k)$.

From Table 2 we see that Theorem 3 holds if $2 \leq k \leq 92$. QED.

THEOREM 4. For $19 \leq \mathrm{k} \leq 286663$ then

$d(k)<k /(\log k-\log \log k)$.

PROOF. To prove this Theorem we must divide this proof into four cases.

Case 1. $6036<k \leq 286663$

Case 2. $388<\mathrm{k} \leq 6036$

Case 3. $193<k \leq 388$

Case $4 . \quad 19<\mathrm{k} \leq 193$

In Cases 1-3 we will need to use the Pascal program called

Verification which is found in section 3, whereas for Case 4 we will use Table 1 .

Case 1. We have

$$
k<2 p_{n}-p_{n+1}
$$

By using (1.3) we see that (2.19) will now become a more restrictive inequality

$$
k<n(\log n+\log \log n-1.5)+p_{n}-p_{n+1}
$$

If we let $n=k /(\log k-\log \log k)$ and we use (1.6) then (2.20) becomes even more restrictive.

$$
k<n(\log n+\log \log n-1.5)-652 .
$$

By using the Pascal program, (2.21) is true for integer $k \in(6036,286663]$ and hence (2.19) is true.

Case 2. Similar to Case 1 up to (2.20). However, we note that if $n=k /(\log k-\log \log k)$ then $n \in[93,922]$. Hence for $n$ in

this range we have $p_{n+1}-p_{n} \leq 34$.

Hence in this range $(2.20)$ becomes

$$
k<n(\log n+\log \log n-1.5)-34
$$

By using the Pascal program we can verify that (2.22) is true and hence (2.19) is true for $k \in(388,6036)$.

Case 3. Similar to Case 2 except that $n \in[54,92]$ and 
$p_{n+1}-p_{n} \leq 14$

Case 4. We look at Table 1 .

IEMMA 7. Let $\mathrm{k} \geq 286664, \mathrm{c}=2.531$ and

$y=(\log (\log (k)-c)-c) /(\log (k)-c)$, then the maximum value of $y$ is approximately .0292756256.

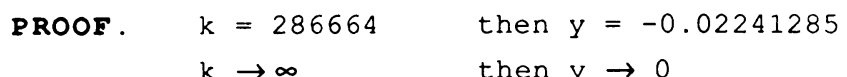

Using elementary calculus we set $y^{\prime}=0$ an see that $k=\exp \left(e^{c+1+c}\right)$ For this $k$ we note that $y^{\prime \prime}<0$ and hence we have a relative maximum; the value of $y$ was determined by using a symbolic language called Maple.

QED .

LEMMA 8. If $k \geq 286664$ and $c=2.531$, let $n=k /(\log (k)-c)$ then $k<n(\log n+\log \log n-2.500539232)$.

PROOF. Suppose not; then

$$
k \geq n(\log n+\log \log n-2.500539232) .
$$

After several manipulations of (2.23) we now have

$0 \geq \log \log (k /(\log (k)-c))-\log (\log (k)-c)+c-2.500539232 .(2.24)$

By grouping the terms containing log functions we see after several steps that (2.24) becomes

$0 \geq \log (1+(c-\log (\log (k)-c)) /(\log (k)-c))+c-2.500539232 . \quad(2.25)$

By Lemma 7 we see immediately that the smallest possible value for the second term within the outer log function is approximately .970724375 . And hence $(2.25)$ is false thereby establishing the Lemma .

QED .

IEMMA 9. For $\mathrm{n} \geq 28567$ then

$2 p_{n}-p_{n+1}>n(\log n+\log \log n-2.500539232)$.

PROOF. Using (1.3) and (1.4) we have

$$
\begin{array}{rl}
2 p_{n}-p_{n+1}>2 & 2(\log n+\log \log n-3 / 2) \\
& -(n+1)(\log (n+1)-\log \log (n+1)-1 / 2) .
\end{array}
$$

After several simple algebraic manipulations and using (1.7), (2.26) becomes

$$
\begin{aligned}
2 p_{n}-p_{n+1}> & n(\log n+\log \log n-2.500003412) \\
& -n \log (1+1 / n)-\log (n+1)-\log \log (n+1) .
\end{aligned}
$$


THEOREM 5. For $k \geq 286664$ then $d(k)<k /(\log k-2.531)$. PROOF. For $\mathrm{k} \geq 286664$, we want to find an upper bound to $d(k)$ such that for all $n \geq d(k)$ we have

$$
k<2 p_{n}-p_{n+1}
$$

For $\mathrm{n} \geq 28567$ and Lemma 9 we have the following

$$
n(\log n+\log \log n-2.500539232)<2 p_{n}-p_{n+1}
$$

From (2.29) we replace (2.28) with a more restrictive inequality

$$
k<n(\log n+\log \log n-2.500539232)
$$

Choose $n=k /(\log k-2.531)$. Then by Lemma $8,(2.30)$ and hence (2.28) still hold. Therefore $d(k)<n=k /(\log k-2.531)$, establishing an upper bound for $d(k)$.

QED .

\section{COMPUTER PROGRAMS AND TABLES.}

The computer program called Verification was written in Turbo Pascal. Tables 1 and 2 were produced by other Pascal programs.

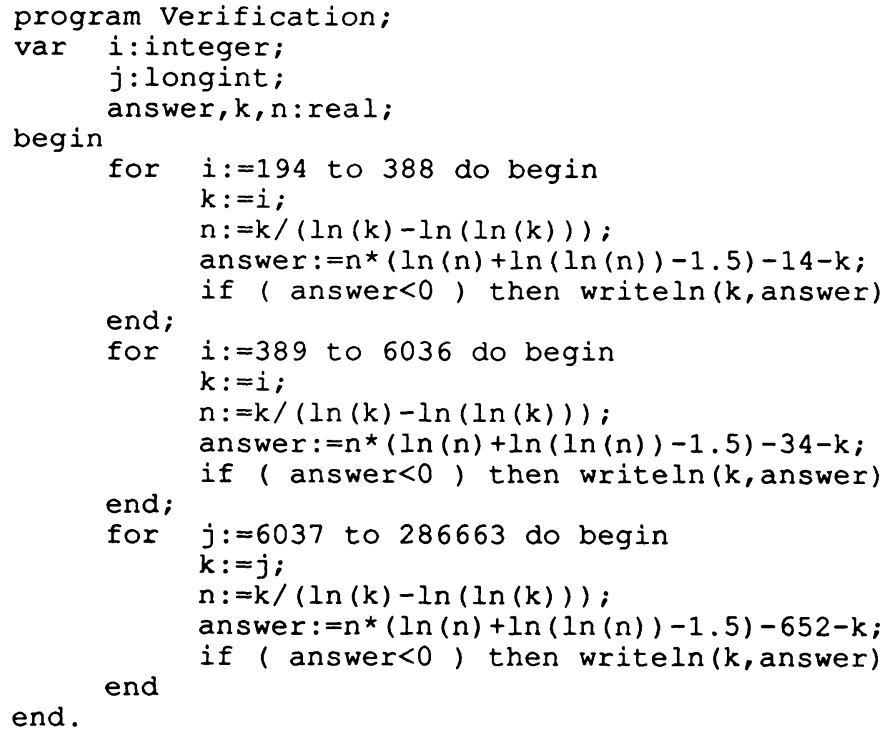


TABLE 1

\begin{tabular}{|c|c|c|c|c|c|c|c|c|c|c|}
\hline k & $d(k)$ & $\mathrm{k}$ & $d(k)$ & $f(k)$ & $k$ & $d(k)$ & $f(k)$ & k & $d(k)$ & $f(k)$ \\
\hline 1 & 3 & 50 & 17 & 19.6235 & 99 & 31 & 32.2462 & 148 & 37 & 43.6793 \\
\hline 2 & 1.8874 & 51 & 17 & 19.9007 & 100 & 31 & 32.4887 & 149 & 37 & 43.9046 \\
\hline 3 & 2.9864 & 52 & 17 & 20.1768 & 101 & 31 & 32.7308 & 150 & 37 & 44.1297 \\
\hline 4 & 3.7748 & 53 & 17 & 20.4519 & 102 & 31 & 32.9723 & 151 & 38 & 44.3545 \\
\hline 5 & 4.4109 & 54 & 17 & 20.7258 & 103 & 31 & 33.2135 & 152 & 38 & 44.5790 \\
\hline 6 & 4.9646 & 55 & 19 & 20.9987 & 104 & 31 & 33.4542 & 153 & 38 & 44.8032 \\
\hline 7 & 5.4680 & 56 & 19 & 21.2706 & 105 & 31 & 6944 & 154 & 38 & 45.0272 \\
\hline 8 & 5.9376 & 57 & 19 & 21.5415 & 106 & 31 & 33. & 155 & 38 & 45.2510 \\
\hline 9 & 6.3828 & 58 & 19 & 21.8115 & 107 & 31 & 34.1737 & 156 & 38 & 45.4745 \\
\hline 10 & 6.8094 & 59 & 19 & 22.0805 & 108 & 31 & 34.4127 & 157 & 38 & 6977 \\
\hline & 7.2211 & 60 & 19 & 22.348 & 109 & 31 & 34.6513 & 158 & 38 & 9207 \\
\hline 12 & 7.6206 & 61 & 19 & 22.61 & 110 & 31 & 34.8894 & 159 & 39 & \\
\hline 13 & 8.0098 & 62 & 19 & 22.8820 & 111 & 31 & 35.1272 & 160 & 39 & 46. \\
\hline 14 & 8.3901 & 63 & 20 & 23.1 & 112 & 31 & 35.3646 & 161 & 4 & 5881 \\
\hline 15 & 8.7626 & 64 & 20 & 23.41 & 113 & 31 & 35.60 & 162 & 4 & 8101 \\
\hline 16 & 9.12 & 65 & 20 & 23.6758 & 114 & 31 & 82 & 163 & 40 & 0319 \\
\hline 17 & 9.48 & 66 & 20 & 23.9387 & 115 & 31 & 4 & 164 & 40 & 47.2534 \\
\hline 18 & 9.8415 & 67 & 22 & 24.2009 & 116 & 31 & 3 & 165 & 40 & 47.4746 \\
\hline 19 & 10.1903 & 68 & 22 & 24.4623 & 117 & 31 & & 166 & 40 & 47.6957 \\
\hline 20 & 10.5344 & 69 & 22 & 24.7229 & 118 & 31 & & 167 & 41 & 47. \\
\hline 21 & 10.8742 & 70 & 22 & 24.9828 & 119 & 31 & .01 & 168 & 41 & 48. \\
\hline 22 & 11.2100 & 71 & 22 & 25.2 & 120 & 31 & .24 & 169 & 41 & 48. \\
\hline 23 & 11.5421 & 72 & 22 & 25.5 & 121 & 31 & 37.4839 & 170 & 41 & 48. \\
\hline 24 & 11.8707 & 73 & 22 & 25.75 & 122 & 31 & 37.7176 & 171 & 4. & 48. \\
\hline 25 & 12.1961 & 74 & 22 & 26.0 & 123 & 32 & 37.9509 & 172 & 43 & \\
\hline 26 & 12.51 & 75 & 23 & 26.27 & 124 & 32 & 38.18 & 173 & 43 & 49. \\
\hline 27 & 12.83 & 76 & 23 & 26.5271 & 125 & 33 & 38 & 174 & 43 & 49.4557 \\
\hline 28 & 13.15 & 77 & 24 & 26.7821 & 126 & 33 & 30 & 175 & 43 & 5747 \\
\hline 2 & 13.46 & 78 & 24 & 27.0365 & 127 & 33 & 6 & 176 & 43 & 49.8 \\
\hline & 3.78 & 79 & 24 & 27.2902 & 128 & 33 & & 177 & 43 & 120 \\
\hline 31 & 14.08 & 80 & 24 & 27.54 & 129 & 35 & 5 & 178 & 43 & 3303 \\
\hline 32 & 4.39 & 81 & 25 & 27. & 130 & 35 & & 179 & 43 & 50 \\
\hline 33 & 4.7010 & 82 & 25 & 28. & 131 & 35 & 39 & 180 & 43 & 64 \\
\hline 34 & 5.0038 & 83 & 25 & 28.2 & 132 & 35 & 40.0354 & 181 & 43 & \\
\hline 35 & 5.3046 & 84 & 25 & 28. & 133 & 35 & 40.26 & 182 & 43 & 51. \\
\hline 3 & 13 & 8 & 25 & 28.7 & 134 & 35 & 40.49 & 183 & 43 & 51 \\
\hline 3 & 13 & 8 & 25 & 29.0 & 135 & 35 & 40.72 & 184 & 4 & 51. \\
\hline 38 & 13 & 8 & 25 & 29.2 & 136 & 35 & 40.95 & 185 & 4 & \\
\hline 30 & 15 & 88 & 25 & 29.5 & 137 & 35 & 41.18 & 186 & 4 & \\
\hline & 15 & & 25 & 29.7 & 138 & 35 & 41.4 & 187 & 47 & \\
\hline & 17.0721 & 90 & 25 & 30.0423 & 139 & 35 & 41.6 & 188 & 47 & \\
\hline & 17.3611 & 91 & 25 & 30.289 & 140 & 35 & 41.86 & 189 & 47 & 52.7183 \\
\hline & 17.6485 & 92 & 25 & 30.5356 & 141 & 35 & 42.0943 & 190 & 47 & 52.9342 \\
\hline & 17.9346 & 93 & 26 & 30.781 & 142 & 35 & 42.3216 & 191 & 47 & 499 \\
\hline 45 & 18.2193 & 94 & 26 & 31.02 & 143 & 35 & 42.5486 & 192 & 47 & 53 \\
\hline 40 & 18.3026 & 95 & 26 & 31.271 & 144 & 35 & 42.7753 & 193 & 47 & .5806 \\
\hline & 17 & 96 & 26 & 31.516 & & 37 & 43.0017 & 194 & 47 & 53.79 \\
\hline 3 & 17 & 97 & 26 & & 146 & 37 & 43.2278 & 195 & 47 & 54.0106 \\
\hline & 17 & 98 & 26 & 32.0033 & 147 & 37 & 43.4537 & & & \\
\hline
\end{tabular}

where $f(k)=k /(\log k-\log \log k)$ 
TABLE 2

$\begin{array}{rrrrrrrrc}k & d(k) & g(k) & k & d(k) & g(k) & k & d(k) & g(k) \\ 1 & 3 & & 32 & 12 & 6.5691 & 63 & 20 & 11.5357 \\ 2 & 3 & -1.2773 & 33 & 13 & 6.7387 & 64 & 20 & 11.6885 \\ 3 & 5 & 0.2451 & 34 & 13 & 6.9075 & 65 & 20 & 11.8410 \\ 4 & 5 & 0.8040 & 35 & 13 & 7.0754 & 66 & 20 & 11.9931 \\ 5 & 5 & 1.1764 & 36 & 13 & 7.2426 & 67 & 22 & 12.1449 \\ 6 & 5 & 1.4797 & 37 & 13 & 7.4090 & 68 & 22 & 12.2963 \\ 7 & 5 & 1.7486 & 38 & 13 & 7.5747 & 69 & 22 & 12.4474 \\ 8 & 5 & 1.9971 & 39 & 15 & 7.7396 & 70 & 22 & 12.5982 \\ 9 & 7 & 2.2319 & 40 & 15 & 7.9039 & 71 & 22 & 12.7487 \\ 10 & 7 & 2.4568 & 41 & 16 & 8.0675 & 72 & 22 & 12.8989 \\ 11 & 7 & 2.6743 & 42 & 16 & 8.2305 & 73 & 22 & 13.0488 \\ 12 & 7 & 2.8858 & 43 & 16 & 8.3929 & 74 & 22 & 13.1984 \\ 13 & 7 & 3.0923 & 44 & 16 & 8.5547 & 75 & 23 & 13.3478 \\ 14 & 7 & 3.2948 & 45 & 16 & 8.7159 & 76 & 23 & 13.4968 \\ 15 & 9 & 3.4936 & 46 & 16 & 8.8766 & 77 & 24 & 13.6455 \\ 16 & 9 & 3.6894 & 47 & 17 & 9.0367 & 78 & 24 & 13.7940 \\ 17 & 10 & 3.8824 & 48 & 17 & 9.1963 & 79 & 24 & 13.9422 \\ 18 & 10 & 4.0730 & 49 & 17 & 9.3554 & 80 & 24 & 14.0902 \\ 19 & 10 & 4.2613 & 50 & 17 & 9.5140 & 81 & 25 & 14.2379 \\ 20 & 10 & 4.4476 & 51 & 17 & 9.6721 & 82 & 25 & 14.3853 \\ 21 & 10 & 4.6320 & 52 & 17 & 9.8297 & 83 & 25 & 14.5325 \\ 22 & 10 & 4.8148 & 53 & 17 & 9.9869 & 84 & 25 & 14.6794 \\ 23 & 10 & 4.9959 & 54 & 17 & 10.1436 & 85 & 25 & 14.8261 \\ 24 & 10 & 5.1756 & 55 & 19 & 10.2999 & 86 & 25 & 14.9726 \\ 25 & 12 & 5.3538 & 56 & 19 & 10.4558 & 87 & 25 & 15.1188 \\ 26 & 12 & 5.5308 & 57 & 19 & 10.6112 & 88 & 25 & 15.2648 \\ 27 & 12 & 5.7065 & 58 & 19 & 10.7663 & 89 & 25 & 15.4105 \\ 28 & 12 & 5.8811 & 59 & 19 & 10.9209 & 90 & 25 & 15.5560 \\ 29 & 12 & 6.0546 & 60 & 19 & 11.0752 & 91 & 25 & 15.7013 \\ 30 & 12 & 6.2271 & 61 & 19 & 11.2291 & 92 & 25 & 15.8464 \\ 31 & 12 & 6.3986 & 62 & 19 & 11.3826 & 93 & 26 & 15.9913\end{array}$

where $g(k)=(k /(\log k))(1-1 / \log k)$

\section{4. coment.}

There is a discrepancy between the value of $d(8)$ found in [4] and the value of $d(8)$ found in this paper. This author believes that the value of $d(8)$ is 5 and not 7 .

\section{ACKNOWLEDGEMENTS .}

I am deeply indebted to Professor J. Repka for his suggestions which led to a better presentation of the manuscript. I would also like to thank the referee for his/her helpful comments. A portion of the cost of this manuscript was supported by the Instructor Development Fund at Ryerson Polytechnic University. 


\section{REFERENCES}

1. SHAPIRO, H.N. Introduction to the Theory of Numbers, John Wiley \& Sons, Inc., New York, 1983, 369-370, 398

2. GIORDANO, G., The Generalization and Proof of Bertrand's Postulate, Internat. J. Math. Math. Sci.10(1987), 821-823

3. DRESSLER, R.E., A stronger Bertrand's postulate with an application to partitions, Proc. Amer. Math. Soc. 33 (1972), 226-228

4. BADEA, C., On a stronger Bertrand's postulate, An. Stiint. Univ. "Al. I. Cuza" Iasi sect. I a Mat. (N.S.) 32 (1986), no. $3,3-5$

5. ROSSER, J.B. and SCHOENEELD, L., Approximate Formulas for Some Functions of Prime Numbers, Ilinois J. Math. 6(1962), 64-94

6. ROSSER, J.B., The $\mathrm{n}^{\text {th }}$ prime is greater than $\mathrm{n}$ log $\mathrm{n}$, Proc. London Math. Soc. (2), 45 (1939), 21-44

7. BRENT, R.P., The First Occurrence of Large Gaps between Successive Primes, Math. Comp._27(1973), 959-963. 


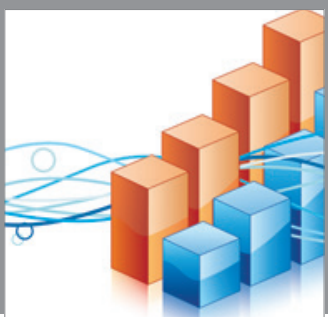

Advances in

Operations Research

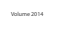

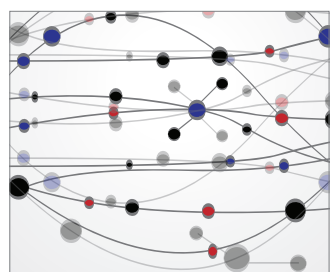

\section{The Scientific} World Journal
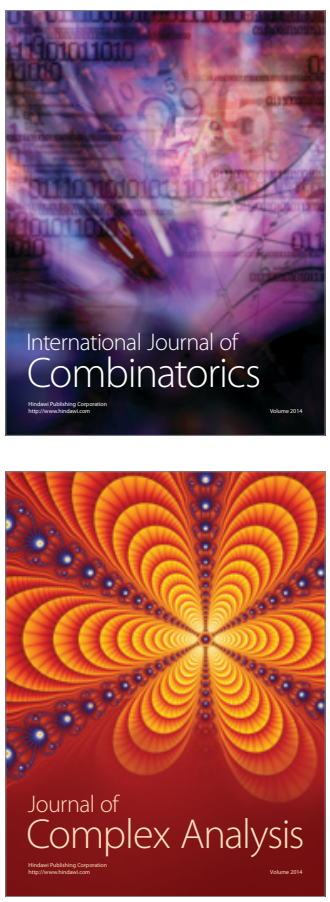

International Journal of

Mathematics and

Mathematical

Sciences
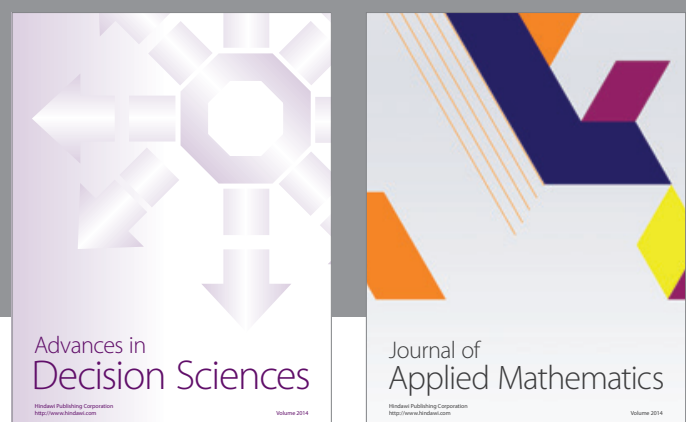

Journal of

Applied Mathematics
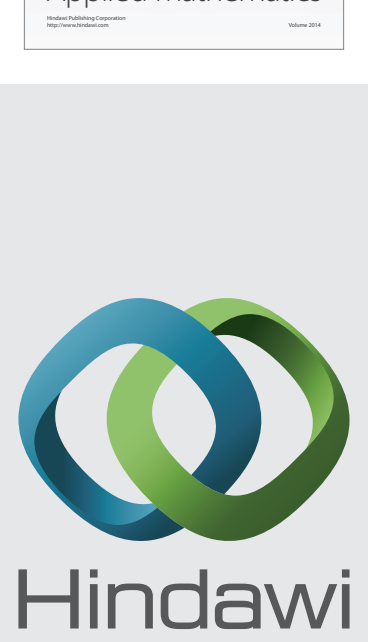

Submit your manuscripts at http://www.hindawi.com
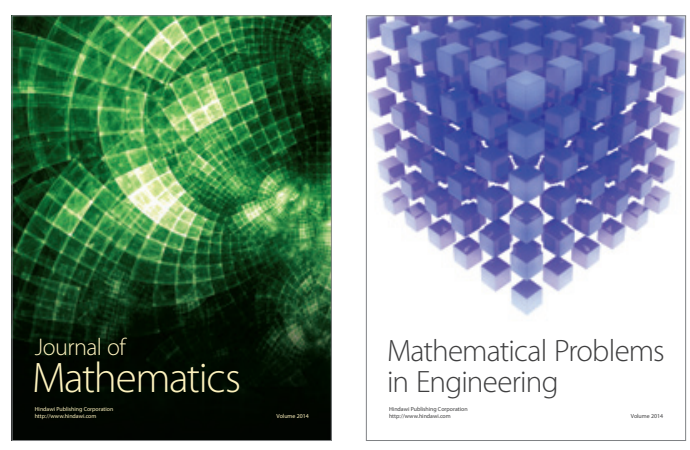

Mathematical Problems in Engineering
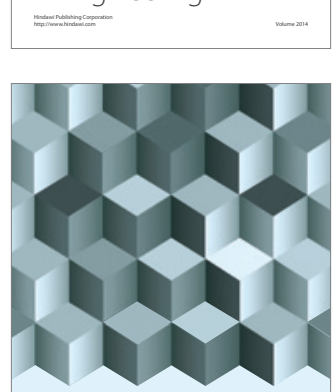

Journal of

Function Spaces
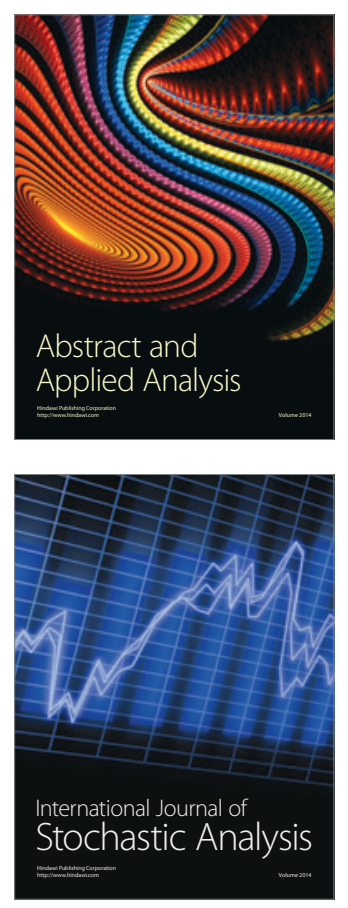

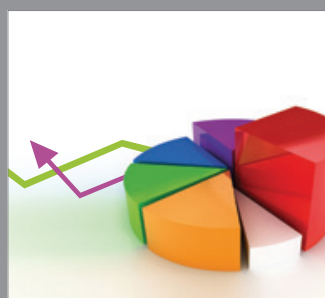

ournal of

Probability and Statistics

Promensencen
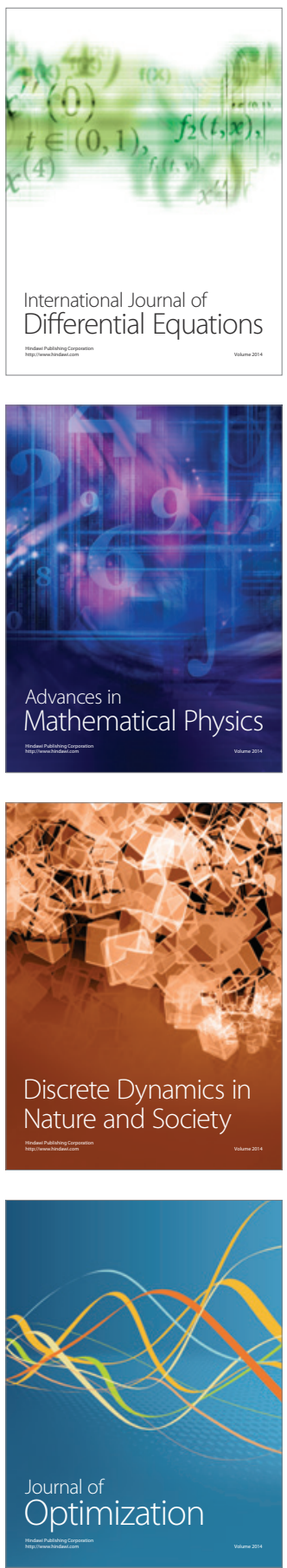